\title{
Citiscreen Cancer Screening: An Update
}

\section{B Petrikovsky*}

\section{Assistant Clinical Professor, Department of Obstetrics, Gynecology and Reproductive} Science, The Mount Sinai Hospital, United States

*Corresponding Author: B Petrikovsky, Assistant Clinical Professor, Department of Obstetrics, Gynecology and Reproductive Science, The Mount Sinai Hospital, United
Received: March 11, 2021

Published: April 23, 2021

(C) All rights are reserved by B Petrikovsky.

States.

CitiScreen puts together fragmented screening system by creating screening algorithms for the following cancers: lung, ovary, breast, prostate, cervix, thyroid colorectal, pancreas, and skin. Individual screening algorithms combine ultrasound, MRI, CT imaging, genetic and tumor markers, as well as other technologies. From the practical point view, screening starts with obtaining family, personal, and social history as well as demographics for identification of risk factors [1].

The decisions to develop, implement and to fund genetic screening programmes are political. Government want to support the biotechnology sector which provides jobs, while on the other hand, governments are also responsible for health services and controlling costs [2]. Current review address key principles of $\mathrm{Ci}$ tiscreen program including its safety.

The following are the modified criteria for individual cancers chosen for screening program:

- There should be a recognizable latent or early symptomatic stage of the disease.

- There should be a suitable test or examination.

- The test should be acceptable to the patients.

- The natural history of the condition, including development from latent to active disease, should be adequately understood.

- $\quad$ There should be a successful treatment strategy leasing to a cure or prolonged survival.

- There should be a defined target population.
- There should be scientific evidence of screening programme effectiveness.

- The programme should integrate education, testing, physical examination as needed and should be updated on the regular basics in view of new scientific developments in the field [3].

\section{Safety considerations}

Recently, a three-dimensional (3D) gradient-echo sequences with isotopic resolution have become available for parenchymal imaging. It takes less than 15 minutes to perform. The TB MRI protocol included an MRI with a detailed examination of the head, neck, chest, abdomen, pelvis, spine and extremities enabling the individual to be covered with coils from "head to toe". Because those examination using MRI or/and CT are to be performed more than once, the safety may become an issue. American Association of Physicists in Medicine states the following: "Risks of medical imaging at effective dose below $50 \mathrm{mSv}$ for single procedures or 100 $\mathrm{mSv}$ for multiple procedures over short time periods are too low to be detectable and may be non-existent." Brenner and Hall published an extensive review on the topic in 2007 New England Journal of Medicine [7]. The radiation doses to particular organs from CT depend on a number of factors. The most important are the numbers of scans, the scanning time in milliamp-seconds (mAs), and the size of the patient, the axial scan range, the tube voltage in the kilovolt peals $[\mathrm{kVp}]$ and the specific design of the scanner [8]. Many of these factors are under the control of the radiologist or radiology technician. Ideally, they should be tailored to the type of study being performed and to the size of the particular patient. Depending on the machine settings, the organ being studied typically receives a radiation dose in the range of 15 milisieverts for

Citation: B Petrikovsky. “Citiscreen Cancer Screening: An Update". Acta Scientific Women's Health 3.5 (2021): 43-44. 
a single CT scan [7]. No large-scale epidemiologic studies of the cancer risks associated with CT scans have been reported; all these concerns had been addressed within Citiscreen projected by the following measures:

- Utilization of the latest generation of scanners with better safety records.

- $\quad$ To replace CT use, when practical, with other options, such as ultrasonography and magnetic resonance imaging (MRI).

\section{Legal considerations}

The choice to perform genetic cancer testing can have legal ramifications for patients and physicians. Patients require informed consent before this test. Physicians and others who are covered by HIPPA are prohibited from disclosing protected health information to third parties without written authorization from the patient [9]. The Health Insurance Portability and Accountability Act contains some limited exceptions permitting disclosure when the covered entity has a good faith belief that the disclose:

- Is it necessary to prevent or lessen a serious and imminent threat to the health or safety of a person,

- Is a person reasonably able to prevent or lessen that threat.

Whether this exception to the privacy rule would include disclosure of genetic test results to a potentially affected family member is uncertain but seems unlikely except in very limited circumstances.

\section{Bibliography}

1. Petrikovsky BM. "New algorithms for cancer screening (CitiScreen project)”. Surgical Oncology 6.2 (2020): 1-3.

2. Knoppers BM., et al. "Commercialization of genetic research and public policy”. Science 286 (1999): 2277-2278.

3. Goel V. "Appraising organized screening programmes for testing for genetic susceptibility to cancer". BMJ 322 (2001): 1174-1178.

4. Rofsky NM., et al. "Abdominal MR imaging with a volumetric interpolated breath-hold examination". Radiology (1999): 876-884.

5. Tarnoki DL., et al. "Clinical value of whole-body magnetic resonance imaging in health screening of general adult population". Radiology Oncology 49.1 (2015): 10-16.
6. American Association of Physicists in Medicine. "AAPM Position Statement on Radiation Risks from Medical Imaging Procedures". Published (2011).

7. Brenner DJ and Hall EJ. "Computed Tomography- An increasing source of radiation exposure". The New England Journal of Medicine 357 (2007): 2277-2284.

8. Brenner DJ. "It is time to retire the computed tomography dose index (CTDI) for CT quality assurance and dose optimization". Medical Physics 33 (2006): 1189-1191.

9. "Legal Considerations in Genetic Screening and Testing: Three Case Studies ACOG Committee Opinion, Number 805". Obstetrics and Gynecology 135.4 (2020): e189-192.

\section{Assets from publication with us}

- Prompt Acknowledgement after receiving the article

- Thorough Double blinded peer review

- Rapid Publication

- Issue of Publication Certificate

- High visibility of your Published work

Website: www.actascientific.com/

Submit Article: www.actascientific.com/submission.php

Email us: editor@actascientific.com

Contact us: +919182824667 